\title{
La première norme mondiale sur les coopératives
}

La recommandation 193/2002 de l'organisation internationale du travail

\section{The first worldwide standard for cooperatives: ILO recommendation 193/2002}

\section{Bruno Roelants}

Numéro 289, juillet 2003

Dossier Coopération

Special Feature on Cooperatives

URI : https://id.erudit.org/iderudit/1022174ar

DOI : https://doi.org/10.7202/1022174ar

Aller au sommaire du numéro

Éditeur(s)

Institut de l'économie sociale (IES)

ISSN

1626-1682 (imprimé)

2261-2599 (numérique)

Découvrir la revue

Citer cet article

Roelants, B. (2003). La première norme mondiale sur les coopératives : la recommandation 193/2002 de l'organisation internationale du travail. Revue internationale de l'économie sociale, (289), 20-29.

https://doi.org/10.7202/1022174ar
Résumé de l'article

Secrétaire général de Cicopa, l'auteur de cet article rend compte du rôle joué par le mouvement coopératif mondial dans l'élaboration de la recommandation $n^{\circ} 193$ de l'Organisation internationale du travail (OIT) concernant la promotion des coopératives (lire l'article de Mark Levin dans ce numéro). Ce sont tout autant la portée de cette nouvelle recommandation que le processus qui a conduit à l'adopter qui sont analysés ici. Comment les organisations coopératives ont-elles pu faire entendre leur voix sur le texte initial, un simple siège d'observateur étant habituellement dévolu à l'Alliance coopérative internationale (ACI) dans le cadre de ces négociations ? Seuls les représentants des gouvernements, des syndicats et des organisations d'employeurs y participent et des accréditations auprès de ces trois groupes étaient nécessaires. Qu'il s'agisse d'obtenir un traitement différencié des coopératives pour ce qui fait leur spécificité par rapport aux autres entreprises ou de souligner la responsabilité des États dans la promotion des coopératives, cette implication du mouvement coopératif a contribué à finaliser un texte réellement utile pour son développement futur. 


\section{La première norme mondiale sur les coopératives}

LA RECOMMANDATION 193/2002 DE L'ORGANISATION INTERNATIONALE DU TRAVAIL ${ }^{(*)}$

(*) Disponible sur le site www.ilo.org de I'OIT. (**) Secrétaire général de Cicopa (Organisation internationale des coopératives de production industrielle, artisanale et de services) e directeur exécutif de Diesis Coop, institution internationale de développement, recherche et formation dans le domaine des coopératives et de l'économie sociale. E-mail : broelants@compuserve.com.

\author{
par Bruno Roelants ${ }^{(*)}$
}

Secrétaire général de Cicopa, l'auteur de cet article rend compte du rôle joué par le mouvement coopératif mondial dans l'élaboration de la recommandation $n^{\circ} 193$ de l'Organisation internationale du travail (OIT) concernant la promotion des coopératives (lire l'article de Mark Levin dans ce numéro). Ce sont tout autant la portée de cette nouvelle recommandation que le processus qui a conduit à l'adopter qui sont analysés ici. Comment les organisations coopératives ont-elles pu faire entendre leur voix sur le texte initial, un simple siège d'observateur étant habituellement dévolu à l'Alliance coopérative internationale (ACI) dans le cadre de ces négociations? Seuls les représentants des gouvernements, des syndicats et des organisations d'employeurs y participent et des accréditations auprès de ces trois groupes étaient nécessaires. Qu'il s'agisse d'obtenir un traitement différencié des coopératives pour ce qui fait leur spécificité par rapport aux autres entreprises ou de souligner la responsabilité des Etats dans la promotion des coopératives, cette implication du mouvement coopératif a contribué à finaliser un texte réellement utile pour son développement futur.

e 20 juin 2002, le concept de coopérative a, pour la première fois depuis son origine au début du XIX siècle, été reconnu pleinement, officiellement, et sans équivoque dans le monde entier, avec tous les paramètres nécessaires et suffisants. Ce jour-là a été votée la recommandation concernant la promotion des coopératives de l'Organisation mondiale du travail (OIT), reprenant exactement la définition, les principes et les valeurs de la déclaration sur l'identité coopérative de l'Alliance coopérative internationale (ACI) adoptée en 1995 à Manchester par le mouvement coopératif. En d'autres termes, les normes internes du mouvement coopératif concernant le concept de coopérative sont devenues des normes officielles au niveau inter-gouvernemental.

Par ailleurs, par l'intermédiaire de cette recommandation, les gouvernements, les syndicats et les entrepreneurs de la majorité des pays du monde ont explicitement reconnu la nécessité de politiques publiques spécifiques pour la promotion des coopératives. 
(1) Disponible sur le site www.ilo.org de l'OIT.
Le vote a été pratiquement unanime: 436 votes pour et aucun vote contre. Seuls se sont abstenus le gouvernement de l'Australie (tandis que 128 gouvernements ont voté favorablement) et les entrepreneurs du Venezuela (tandis que les entrepreneurs de 94 pays ont voté favorablement). Même si 46 gouvernements membres de l'OIT étaient absents lors du vote, les gouvernements de tous les pays grands et moyens, qui bénéficient d'une certaine influence sur la politique de leurs régions respectives, ont voté. Au niveau européen, tous les pays membres de l'UE ainsi que les pays en phase d'accession et candidats (28 Etats au total) ont voté en faveur de la recommandation. Hormis la convention et la recommandation sur les pires formes de travail infantile (1999), qui ont été votées à l'unanimité, la recommandation 193 est l'instrument de l'OIT qui a atteint le plus haut niveau de consensus au moins depuis 1997.

Une autre caractéristique tout à fait unique à cette recommandation est qu' elle contient une annexe qui cite un texte provenant d'une organisation (l'ACI) ne faisant pas partie des Nations unies, et qui mentionne même le nom de cette organisation. C'est la première fois depuis sa création au début du XXe siècle que l'OIT cite une organisation externe au système des Nations unies dans un de ses textes officiels.

Par conséquent, sous différents angles (normes internes devenues officielles, reconnaissance explicite des acteurs concernés et de leur organisation mondiale, reconnaissance explicite de la nécessité de les promouvoir, consensus obtenu), cette recommandation se distingue clairement de la plus grande partie des textes officiels de l'OIT.

Nous examinerons ci-dessous les points saillants du contenu de la recommandation, y compris ce qui la distingue d'une précédente recommandation, qui ne s'appliquait qu'aux pays en développement (recommandation 127 de $1966^{(1)}$ ). Ensuite, nous verrons comment ce résultat a été obtenu, lors des semaines de négociations et des années de préparation du résultat final, et surtout l'apport du mouvement coopératif lui-même à ce résultat. Nous terminerons par une conclusion provisoire, concernant les opportunités que cette recommandation offre pour le mouvement coopératif.

\section{Principaux acquis de la recommandation et ce qu'aurait signifié leur contraire}

La recommandation est explicitement universelle, tandis que la précédente recommandation sur les coopératives, qui, comme nous venons de le voir, ne s'appliquait qu'aux pays en développement, était centrée avant tout sur les coopératives agricoles. Elle s'adresse à toutes les formes de coopératives, qui sont reconnues comme pouvant opérer dans tous les secteurs de l'économie. Le fait que la précédente recommandation n'était pas universelle en limitait fortement l'intérêt politique.

Le texte reprend la définition de coopérative dans sa forme originale, ainsi que les dix valeurs coopératives et les sept principes coopératifs avec 
leur explication originale dans une annexe, tout en mentionnant la source (la déclaration sur l'identité coopérative de l'ACI, Manchester, 1995). La précédente recommandation, qui contenait une définition sui generis où n'apparaissaient pas les principes et valeurs coopératifs et qui ne s'appliquait qu'à certains pays, n’avait pas cette dimension normative. Ce fait est d'une importance stratégique fondamentale, pour deux raisons. D'abord parce que le mouvement coopératif voit ses propres normes officialisées: si d'autres normes avaient été adoptées (ce qui a failli advenir, lire la section suivante), le mouvement coopératif aurait dû, pour maintenir son identité, se battre pendant de longues années contre un cadre normatif mondial différent du sien. Ensuite parce que, de cette façon, le mouvement coopératif est ouvertement reconnu (non seulement par les gouvernements, mais aussi par les représentants des travailleurs et des employeurs) comme un acteur socio-économique mondial qu'il convient de respecter dans l'élaboration de ses propres normes. Il s'agit ici d'un important précédent pour prévenir que d'autres forces socio-économiques ne tentent d'imposer leurs propres normes aux coopératives.

Sur la base des normes spécifiques des coopératives (lire le point ci-dessus), la recommandation reconnaît que les coopératives sont des entreprises spécifiques qui requièrent un traitement spécifique. Alors que la précédente recommandation reconnaissait la spécificité de la forme coopérative, les différents textes préparatoires précédant la version finale de la nouvelle recommandation contenaient des références, au contraire, à la nécessité de traiter les coopératives comme toute autre forme d'entreprise. Si cette version avait été adoptée (ce qui fut à deux doigts d'advenir), le mouvement coopératif aurait probablement dû pendant longtemps se battre pour s'assurer que les coopératives continuent à obtenir un traitement différencié pour tous les aspects qui intrinsèquement les différencient d'autres espèces d'entreprises. Toute tentative de standardisation totale des coopératives à l'aune des entreprises conventionnelles aurait été dès lors facilitée. La contribution des coopératives à la société et aux objectifs des politiques publiques reçoit une reconnaissance particulièrement forte. Les coopératives "sous leurs différentes formes promeuvent la plus complète participation au développement économique et social de toute la population " (préambule) et contribuent à des "objectifs des politiques sociales et publiques tels que la promotion de l'emploi ou la mise en oeuvre d'activités qui s'adressent aux groupes ou régions défavorisés» (art. 7.2). Elles sont bien des "entreprises et organisations inspirées par l'esprit de solidarité " (art. 5), qui doivent " répondre aux besoins de leurs adhérents et de la société, y compris à ceux des groupes défavorisés afin de les insérer dans la société" " (ibid.). Tous ces concepts sont innovants par rapport à la précédente recommandation.

Sur la base de ce constat, les coopératives doivent non seulement bénéficier d'un cadre de régulation différencié, mais aussi être promues activement par des "mesures particulières" (art. 5). Le texte va jusqu'à dire que cette promotion "devrait être considérée comme l'un des piliers du développement économique et social national et international " (art. 7.1). 
Il est également clarifié que ce sont les gouvernements qui sont les principaux responsables de la promotion des coopératives. En effet, les gouvernements sont mentionnés à neuf reprises, c'est-à-dire dans pratiquement tous les articles se référant aux politiques de promotion des coopératives. Dans plusieurs versions provisoires du texte, ces phrases étaient à la forme passive, laissant planer le doute concernant la responsabilité des Etats dans la promotion des coopératives. Si cette forme passive avait été maintenue, ce qui était sur le point d'advenir, le rôle et les responsabilités de l'Etat n'auraient pas été soulignés et, en l'absence d'un acteur clairement défini responsable de la promotion des coopératives, cette recommandation aurait perdu une grande partie de son intérêt concret.

Le texte argumente que l'éducation coopérative devrait faire partie de l'enseignement formel et régulier. En effet, " les politiques nationales devraient [...] promouvoir l'enseignement des principes et pratiques coopératifs et la formation [...], à tous les niveaux appropriés des systèmes nationaux d'éducation et de formation et dans l'ensemble de la société " (art. 8.1 e). Cette phrase, généralement inaperçue dans le texte, est riche en potentiel de politiques publiques. Il signifie notamment que les programmes et manuels scolaires devraient inclure une introduction au fonctionnement d'une coopérative. Une référence similaire existait dans la précédente recommandation, mais en mettant l'accent sur l'enseignement technique et professionnel et se référant uniquement aux pays en développement.

Les organisations représentatives des coopératives apparaissent comme le quatrième acteur principal dans la recommandation, à côté des gouvernements, des syndicats et des organisations d'employeurs, les trois corps constituants de l'OIT. Ces quatre acteurs devraient collaborer " en vue de créer un climat favorable au développement des coopératives ». De plus, les organisations coopératives se voient invitées à "représenter le mouvement coopératif national au niveau international ", ce qui est clair au sein du mouvement coopératif, mais ne l'est pas forcément au niveau des instances internationales externes à celui-ci (art. 17). Même si les fédérations coopératives étaient mentionnées dans la précédente recommandation, elles étaient loin d'y avoir une telle position.

La recommandation fait référence à la promotion de structures de développement coopératif, tant internes au mouvement coopératif que publiques ou parapubliques. En effet, "les gouvernements devraient reconnaître le rôle des coopératives et de leurs organisations en développant des instruments appropriés destinés à créer et à renforcer les coopératives aux niveaux local et national» (art. 11.4). Mais il ne s'agit pas seulement de services d'appuis externes, puisque les organisations coopératives " devraient être encouragées à $[\ldots]$ gérer leurs propres services d'appui et contribuer à leur financement " (art. 17 b). Or, l'expérience du mouvement coopératif montre que l'une des clés du développement de ce dernier a

(2) Roelants B. (2000), " Worker cooperatives and socio-economic development : the role of mesolevel institutions", Economic Analysis, vol. 3, $n^{\circ} 1$. été depuis plus de cent ans l'existence de telles structures de niveau "méso » (2). Ce point-ci est complètement innovant par rapport à la précédente recommandation. 
L'importance des réserves impartageables et des fonds mutuels est soulignée, même si légèrement moins fort que ce que nous avions proposé: «Les gouvernements devraient mettre en place une politique et un cadre juridique favorables, conformes à la nature et à la fonction des coopératives et fondés sur les valeurs et principes coopératif $[\ldots]$ visant à [...] promouvoir des politiques ayant pour but de permettre la constitution de réserves appropriées, dont une partie au moins pourrait être indivisible, et de fonds de solidarité au sein des coopératives " (art. 6 a). Ces concepts étaient totalement absents de la recommandation de 1966.

La recommandation indique que les principales normes du travail doivent s'appliquer également aux travailleurs des coopératives (préambule), tout en reconnaissant que les organisations syndicales devraient "promouvoir l'exercice des droits des travailleurs associés des coopératives" (art. 16 g). La précédente recommandation ne mentionnait ni les normes fondamentales du travail ni le travail associé.

Une autre innovation est le concept d'économie sociale, sans que le texte mentionne toutefois nommément cette dernière. "Des mesures devraient être adoptées pour promouvoir le potentiel des coopératives dans tous les pays, quel que soit leur niveau de développement, afin d'aider celles-ci et leurs adhérents $\grave{a}[\ldots]$ créer et développer un secteur bien particulier de l'économie, viable et dynamique, comprenant les coopératives, qui répond aux besoins sociaux et économiques de la collectivité" (art. 4). Par ailleurs, "l'équilibre d'une société exige quil existe des secteurs public et privé puissants, ainsi qu’un puissant secteur coopératif, mutualiste et autres organisations sociales et non gouvernementales. C'est dans ce contexte que les gouvernements devraient mettre en place une politique et un cadre juridique favorables, conformes à la nature et à la fonction des coopératives et fondés sur les valeurs et principes coopératifs [...] " (art. 6). La coopération internationale est mentionnée comme dans l'ancienne recommandation, mais avec davantage de points concrets, comme les échanges de savoir-faire et d'expérience, les partenariats et l'accès à l'information (art. 18). De plus, la recommandation mentionne l'opportunité de développer des "directives et des législations régionales et internationales communes favorables aux coopératives " (art. $18 d$ ). Deux semaines avant le vote sur la nouvelle recommandation, le Conseil de l'Union européenne venait justement d'approuver les statuts de la société coopérative européenne, le tout premier texte législatif supra-national sur les coopératives.

\section{Comment ces résultats ont-t-ils été obtenus? Le rôle des organisations coopératives dans la négociation}

Les résultats mentionnés dans la précédente section n'ont pas été obtenus facilement. Le mouvement coopératif mondial a fait des efforts importants pour qu'ils adviennent, dans un contexte où, en principe, il n'a pas de place dans les négociations, mais n'est admis comme tel que de façon passive, avec un siège d'observateur attribué à l'ACI. En effet, les négociations de 
(3) Cecop 2001, "Première recommandation mondiale sur les coopératives ", mimeo, disponible auprès de cecop@ceop.org
l'OIT ne se déroulent qu'entre ses membres constitutifs, qui sont les gouvernements, les syndicats et les organisations patronales des différents pays du monde. Il est important de retracer brièvement l'histoire de ce processus du point de vue des organisations coopératives, parce qu'elle montre comment un acteur organisé de l'économie sociale peut influer sur une négociation mondiale qui le concerne au premier degré. Ce résultat est dès lors une partie intrinsèque du sens et de la portée de la recommandation. La mobilisation au sein des diverses organisations (nationales, régionales, internationales) du mouvement coopératif concernant la recommandation a commencé début 2000 , un an et demi avant la première session de négociations.

Dès le début, le Cicopa, l'organisation spécialisée de l'Alliance coopérative internationale pour les coopératives de production (les Scop) et pour les coopératives sociales, ainsi que ses organisations régionales et nationales ont joué un rôle clé dans cette mobilisation. En effet, il ressortait de la première enquête de l'OIT sur cette recommandation que la principale motivation des pays membres pour une recommandation sur les coopératives était dès le début l'emploi et l'inclusion sociale, domaines qui touchent de très près les Scop et les coopératives sociales.

La Confédération européenne des coopératives de travail associé, des coopératives sociales et des entreprises participatives (Cecop), organisation régionale du Cicopa, a d'abord procédé à une première analyse critique des résultats de l'enquête préliminaire de l'OIT et du premier texte proposé sur la base de cette enquête ${ }^{(3)}$.

Très rapidement, un certain nombre d'organisations nationales intersectorielles de coopératives, contactées par le Cicopa et ses organisations régionales et nationales, se sont également mobilisées sur ce dossier et, peu avant la première session de négociations, ont formulé des positions nationales (par exemple, France, Italie, Espagne, République tchèque, Brésil). Ces positions nationales montraient de nombreux points de convergence, notamment en ce qui concerne le traitement spécifique des coopératives et le rôle de l'Etat.

Cependant, il était apparu dès le début indispensable que les organisations coopératives ne limitent pas leur apport à la rédaction de positions écrites, mais qu'elles puissent participer directement aux travaux d'élaboration de la recommandation. Or, les seules parties prenantes aux travaux de l'OIT sont les représentants des gouvernements, des syndicats et des organisations d'employeurs, les trois seules parties constitutives de l'organisation. Plusieurs représentants d'organisations coopératives, y compris l'auteur de cet article, se sont donc fait accréditer auprès de délégations nationales à la $89^{\circ}$ Conférence internationale du travail (2001), soit dans le groupe des travailleurs, soit dans celui des employeurs, soit dans celui des gouvernements. Nous étions finalement une douzaine de représentants d'organisations coopératives, répartis entre les trois parties constitutives de l'OIT, au sein d'une commission spécialisée sur les coopératives, comprenant plus de cent cinquante personnes. 
Dès le premier jour des négociations, notre groupe coopératif, enrichi par quelques représentants « amis » de gouvernements comme ceux de l'Espagne et du Brésil, a dû très rapidement apprendre comment fonctionnaient les règles de la négociation à l'OIT. Par exemple, nous avons dû en l'espace de quelques heures rédiger des propositions d'amendement, donc obtenir un consensus entre nous tous qui représentions trois langues différentes, sans interprètes et en travaillant dans les couloirs. Cette situation nous distinguait des groupes organisés et reconnus institutionnellement, comme celui des travailleurs - qui avait déjà préparé des dizaines d'amendements à l'avance -, et qui disposaient de toutes les infrastructures et interprètes nécessaires pour finaliser leurs rédactions.

Sur 177 amendements introduits pendant la première session de négociations, le groupe coopératif en a introduit 16, dont la moitié ont été approuvés, après les avoir expliqués en commission et en avoir discuté avec les différents groupes dans des réunions ad hoc.

A la fin de la première session de deux semaines, en 2001, le texte sur lequel nous travaillions s'était déjà nettement amélioré. Presque toutes les références aux limites du rôle de l'Etat et à l'égalité totale entre coopératives et autres espèces d'entreprises, soutenues avant tout par le groupe des employeurs, avaient été éliminées. Cependant, le texte était loin d'être le produit final que nous souhaitions obtenir. Notamment, nous n'étions pas arrivés à obtenir la définition de la coopérative de l'ACI, et encore moins la déclaration d'identité coopérative (Manchester, 1995) dans son ensemble. Le texte provisoire ne contenait encore aucune référence aux réserves impartageables ni au travailleur associé. Nous avions une année pour essayer d'atteindre le consensus le plus large possible au sein du mouvement coopératif mondial et pour nous préparer le mieux possible à la dernière session de quinze jours. Nous présumions également que le groupe des employeurs et une partie des gouvernements des pays industrialisés travailleraient entretemps dans un sens différent.

Nous avons travaillé sur un consensus en "boule de neige ". Un premier groupe de vingt-trois organisations coopératives européennes et latinoaméricaines ont demandé et obtenu que la recommandation soit mentionnée dans une résolution de l'assemblée générale de l'ACI d'octobre 2001, prévoyant un groupe de travail ad hoc. La Confédération européenne des coopératives de travail associé et sociales (Cecop, partie européenne du Cicopa) a approuvé une proposition de texte modifié, que le Cicopa a à son tour approuvée, en accord avec les représentants coopératifs qui étaient venus à Genève à la première session de la négociation. Les organisations coopératives du Costa Rica ont ensuite organisé en mars 2002, sous les auspices de l'ACI-Amérique, une conférence panaméricaine sur la recommandation: les organisations coopératives d'Amérique latine et des Caraïbes y ont approuvé un texte basé sur celui du Cicopa, enrichi de nouvelles propositions. Ce texte a ensuite été examiné et légèrement enrichi en avril 2002 par le groupe de travail institué par l'ACI, qui comprenait des membres du conseil d'administration de cette dernière ainsi que des représentants des 
différentes parties prenantes dans le processus de consultation: Cicopa, organisations inter-coopératives d'Amérique latine et des Caraïbes, de France et d'Italie. Ce dernier texte, avec des propositions provenant de plus de trente organisations coopératives nationales, régionales et sectorielles, sur les cinq continents, a été approuvé fin avril par le conseil d'administration de l'ACI.

Nous arrivions donc à la dernière session de négociations, en juin 2002, avec une position construite avec la participation d'une masse critique d'organisations coopératives, et légitimée par l'organisation représentant le mouvement coopératif au niveau mondial. Par ailleurs, nous connaissions nettement mieux le terrain et les règles des négociations de l'OIT que l'année précédente. Mais la discussion est apparue dès le début comme étant beaucoup plus polarisée que la première fois. Les gouvernements des pays industrialisés et les employeurs ont essayé de rediscuter la question du traitement différencié des coopératives et du rôle de l'Etat, qui avaient pourtant été négociées l'année précédente. En revanche, notre groupe coopératif recevait cette fois l'appui explicite de pratiquement tous les gouvernements africains et latino-américains représentés en commission, ainsi que celui des syndicats. Dans ces deux blocs en présence, le nôtre offrait un très léger avantage numérique. Cependant, le risque de ne pas arriver à une recommandation construite par consensus aurait largement détruit cet avantage. En effet, la recommandation n'aurait pas eu la même force politique si elle avait été désapprouvée par des gouvernements de pays industrialisés importants et par l'ensemble des organisations d'employeurs, ce qui est arrivé dans plusieurs recommandations de l'OIT ces dernières années.

La difficulté a donc été de faire passer au deuxième plan le phénomène des deux « blocs » qui était apparu et de travailler le plus possible selon la logique du consensus. Le résultat de cette stratégie a été tangible : $63 \%$ des 47 amendements que nous avions introduits (le tiers de tous les amendements introduits), basés sur le consensus atteint au sein du mouvement coopératif, ont été approuvés.

\section{Conclusion provisoire: à quoi peut servir concrètement la recommandation 193 ?}

Ce nouvel instrument de l'OIT est utile en tant que tel comme texte normatif dans toute négociation entre les organisations coopératives de niveau national et régional et les autorités publiques de même niveau. Même s'il ne s'agit que d'une recommandation, les gouvernements de la plupart des pays du monde l'ont approuvée activement: en cas de législation ou politique contraire proposée par les autorités d'un pays ou d'un groupe de pays liés par un système d'intégration régionale, le mouvement coopératif correspondant est en mesure de rappeler à ces autorités qu'elles agissent de façon contraire à ce qu'elles ont précédemment voté. D'autant plus que 
(4) C'est notamment ce qu'a proposé un expert de la Banque interaméricaine de développement à la dernière conférence de celle-ci, à Milan en mars 2003.

(5) En contradiction totale avec les preuves historiques, qui montrent que le mouvement coopératif a dans la plupart des cas démarré longtemps avant le système communiste.
l'OIT exerce maintenant un certain suivi sur ses recommandations, notamment par un rapport demandé aux pays membres dans les dix-huit mois suivant leur adoption.

Cet aspect normatif tend à être valorisé nettement plus par les organisations des pays en développement que par celles des Etats membres de l'UE. En effet, ces derniers vont maintenant disposer d'un début de cadre normatif avec les statuts de la société coopérative européenne, tandis que les autres n'ont aucun autre texte de référence normatif complet au niveau supra-national. Par ailleurs, ces pays sont nettement plus vulnérables au niveau du cadre normatif des coopératives en raison de la pression exercée par les institutions financières internationales, qui vont parfois jusqu'à prôner ouvertement la transformation des coopératives en sociétés anonymes conventionnelles ${ }^{(4)}$.

Dans les pays qui ont connu une économie planifiée de type communiste (notamment huit d'entre eux qui entreront dans l'UE dans moins d'un an) où le mouvement coopératif est erronément accusé de représenter un vestige du système précédent ${ }^{(5)}$ et est également l'objet de tentatives de liquidation pour des raisons idéologiques, le fait que ce texte ait été approuvé par les gouvernements des principaux pays industrialisés (UE, EtatsUnis, Japon, etc.) est également un point de référence important.

Cependant, les pays de l'UE auraient tort de penser que les normes mondiales de l'OIT et la situation mondiale des coopératives passent au deuxième plan par rapport au cadre normatif européen. En effet, la Commission européenne elle-même, dans l'établissement d'un cadre normatif européen pour les coopératives, a suivi de près le processus de construction de cet instrument mondial. De plus, la vulnérabilité des systèmes coopératifs en dehors de l'UE (qui accroît leur intérêt pour la recommandation de l'OIT) est une menace indirecte et à plus long terme, mais bien réelle pour les coopératives dans l'UE elle-même.

En ce qui concerne les politiques proactives de promotion des coopératives, l'effet de la recommandation dépendra de l'orientation des différents Etats concernant l'emploi, la lutte contre la pauvreté et l'exclusion, etc.

La recommandation peut également faciliter les relations des organisations coopératives avec les organisations syndicales et patronales, sachant qu' une majorité d'entre elles l'ont approuvée.

Afin de dégager toute sa potentialité, cependant, la recommandation devra d'abord être examinée par l'OIT elle-même en termes de politiques concrètes. Ce pas est important pour que les autres agences du système des Nations unies en fassent de même et pour que la promotion des coopératives devienne un phénomène significatif au niveau international.

Quoi qu'il en soit, la recommandation 193/2002 de l'OIT sur la promotion des coopératives arrive au moment où le mouvement coopératif est à un tournant de son histoire, avec de nouvelles menaces et de nouvelles opportunités. En effet, les coopératives sont confrontées à des pressions de plus en plus puissantes, dans de nombreuses régions du monde, en faveur de leur disparition. Si la globalisation économique se poursuit, avec moins 
de régulation globale institutionnelle et plus d'initiatives basées sur la force, les crises financières et les conflits armés pourraient largement la mettre en échec. Par contre, si une crise de la coordination des chaînes de production et de commercialisation ainsi que des plus grandes entreprises d'échelle mondiale se produit, de nouvelles réalités coopératives pourront voir le jour. Dans les deux cas, que le mouvement coopératif doive développer une stratégie défensive pour éviter sa propre disparition ou qu'il soit amené à négocier des politiques proactives de promotion face à de nouvelles opportunités, ce nouvel instrument international sur les coopératives peut se révéler particulièrement utile. 